\title{
Температурная зависимость атомной структуры и электрической активности дефектов в термоэлектрике ZnSb, слабо легированном медью
}

\author{
(С) Л.В. Прокофььева ${ }^{1}$, Ф.С. Насрединов ${ }^{2,3}$, П.П. Константинов ${ }^{1}$, А.А. Шабалдин ${ }^{1}$ \\ ${ }^{1}$ Физико-технический институт им. А.Ф. Иоффе Российской академии наук, \\ 194021 Санкт-Петербург, Россия \\ ${ }^{2}$ Санкт-Петербургский политехнический университет Петра Великого, \\ 195251 Санкт-Петербург, Россия \\ ${ }^{3}$ Военная академия связи им. С.М. Буденного \\ 194064 Санкт-Петербург, Россия \\ E-mail: Iprokofieva496@gmail.com
}

(Получена 28 декабря 2016 г. Принята к печати 10 января 2017 г.)

\begin{abstract}
Решается задача выбора модели для описания температурной зависимости микроструктуры дефектов в эффективном термоэлектрике $\mathrm{ZnSb}$ с $0.1 \mathrm{aT} \% \mathrm{Cu}$. Анализируются температурные зависимости концентрации и подвижности для термоцикла 300-700-300 K (термоцикл I) с учетом особенностей кристаллической структуры и ковалентного характера химической связи в ZnSb. Базовой дефектной структурой (температуры $T=560-605 \mathrm{~K}$ ) является состояние, когда все атомы $\mathrm{Cu}$ поровну распределены между узлами обеих подрешеток, являясь акцепторами, собственных дефектов акцепторного и донорного типа значительно меньше. Их действие становится заметным, когда температура выходит за рамки вышеупомянутого диапазона. При $T>605 \mathrm{~K}$ появляются дополнительные акцепторы - антиструктурный цинк $\mathrm{Zn}_{\mathrm{Sb}}$; при охлаждении ниже $560 \mathrm{~K}$ образуются димеры $\mathrm{Cu}_{2}$, электрическая активность примеси понижается. Распад димеров при нагревании вызывает увеличение концентрации с температурой вплоть до насыщения в указанном выше диапазоне. Были проведены дополнительные термоциклы II-VIII, обнаруженные изменения в температурных зависимостях концентрации дырок и подвижности обсуждаются в рамках упомянутой модели.
\end{abstract}

DOI: $10.21883 /$ FTP.2017.09.44878.8510

\section{1. Введение}

Ранее сообщалось, что в легировании материала $\mathrm{ZnSb}$, приготовленного стандартным прессованием с последующим гомогенизирующим отжигом, большую роль играют температурно-зависимые процессы передислокации примесных атомов в решетке, вызывающие значительный, особенно при пониженных температуpax, температурный гистерезис в поведении свойств, связанный с неравновесными состояниями дефектов при измерениях [1]. От этих процессов существенно зависят основные параметры легированного $\mathrm{ZnSb}$ как термоэлектрика, прежде всего концентрация дырок $P$ и их подвижность $\mu$. Исследованиями коэффициентов термоэдс $\alpha$, электропроводности $\sigma$, теплопроводности $\kappa$ в диапазоне температур $T=300-700 \mathrm{~K}$ на образцах $\mathrm{ZnSb}$ разного легирования были определены перспективные составы материала, показавшие величину термоэлектрической эффективности $Z T$ в интервале 0.9-1.1 при температурах 550-700 K [2]. Это позволяет утверждать, что уровень достижений в физике и технологии термоэлектрика $\mathrm{ZnSb}$ в мире растет [3-8].

Этот результат явился решением первой задачи выбора новых термоэлектрических материалов с учетом современных реалий; не менее, а возможно, и более важной является вторая задача - подтвердить, что сохранение свойств при кратковременной выдержке образца при рабочих температурах в процессе измерения не является случайностью, что эффективный материал рассчитан на долгий срок службы при выборе оптимальных условий его использования. Исследования такого характера, касающиеся разных составов $\mathrm{ZnSb}$, и составляют в настоящее время нашу основную цель. В данной работе интерес сосредоточен на природе упомянутых выше процессов и их влиянии на стабильность термоэлектрических свойств легированного материала в зависимости от условий его применения. Для первых исследований выбран эффективный образец $\mathrm{ZnSb}$ с добавкой $0.1 \mathrm{aт} \% \mathrm{Cu}$. Данные по эффекту Холла и электропроводности для термоцикла I в диапазоне 300-700-300 K, ранее полученные в [1], служат основой для анализа и создания адекватной модели описания протекающих процессов в микроструктуре дефектов, которые находят отражение в температурном поведении свойств. Вторая часть работы включает проведение на этом же образце дополнительных термоциклов, обсуждение обнаруженных отличий в температурных кривых $P, \sigma$ и $\mu$, определение оптимальных условий использования данного состава $\mathrm{ZnSb}$ с сохранением высоких значений $Z T$ в сочетании с достаточно высокой стабильностью. 


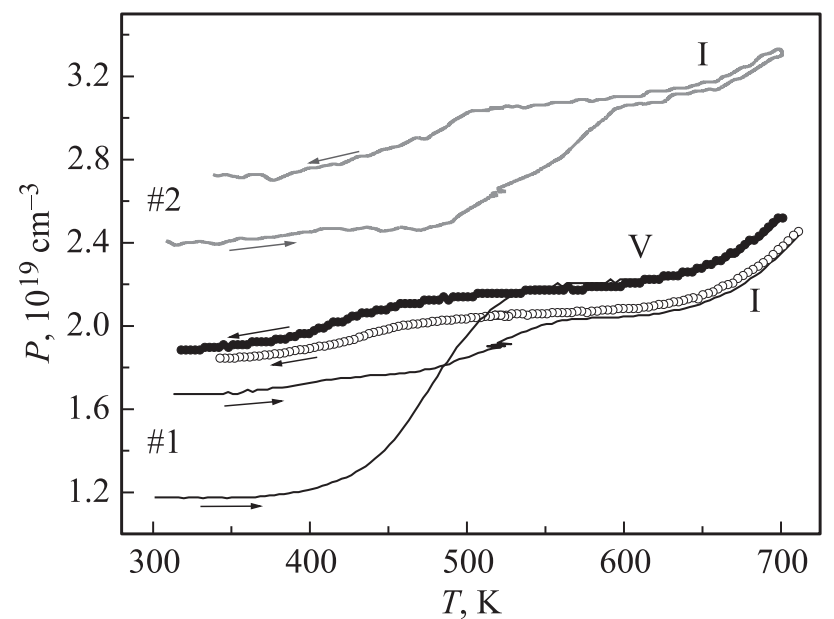

Рис. 1. Температурные зависимости холловской концентрации в образце $\mathrm{ZnSb}: 0.1 \mathrm{aт} \% \mathrm{Cu}(\# 1)$ для циклов $\mathrm{I}$ и $\mathrm{V}$ и в образце $\mathrm{ZnSb}: 0.15 \mathrm{aT} \% \mathrm{Cu}(\# 2)$ для цикла I.

\section{2. Анализ температурных зависимостей холловской концентрации и подвижности в начальном цикле}

На рис. 1 представлена температурная зависимость холловской концентрации дырок $P$ в прессованном и отожженном образце $\mathrm{ZnSb}$ с концентрацией $\mathrm{Cu} 0.1$ ат\% (образец \#1) для цикла I по данным [1]. На кривой имеются 3 участка, и только на одном из них, 560-605 K, постоянная концентрация носителей заряда $P$ равна полной концентрации атомов меди $[\mathrm{Cu}]=2.05 \cdot 10^{19} \mathrm{~cm}^{-3}$. Величина $1 /[\mathrm{Cu}]=5 \cdot 10^{-26} \mathrm{M}^{3}$ заметно превосходит объем элементарной ячейки $\mathrm{ZnSb}$, равный $3.8 \cdot 10^{-28} \mathrm{M}^{3}$, и в этом отношении концентрацию меди можно считать малой.

В общем виде с учетом кристаллической структуры $\mathrm{ZnSb}$ и ковалентного характера химической связи уравнение электронейтральности для $\mathrm{ZnSb}: \mathrm{Cu}$ записывается в виде

$$
\begin{aligned}
P= & N_{A}-N_{D}=\left[\mathrm{Cu}_{\mathrm{Zn}}\right]+\left[V_{\mathrm{Zn}}\right]+\left[\mathrm{Cu}_{\mathrm{Sb}}\right]+\left[\mathrm{Zn}_{\mathrm{Sb}}\right] \\
& -\left[\mathrm{Cu}_{i}\right]-\left[\mathrm{Zn}_{i}\right]-\left[V_{\mathrm{Sb}}\right]-\left[\mathrm{Sb}_{\mathrm{Zn}}\right]+a\left[\mathrm{Cu}_{2}\right],
\end{aligned}
$$

где скобки [] обозначают концентрацию, индексы позицию атома в решетке, акцепторы с $(+)$, доноры c $(-), \mathrm{Cu}_{2}$ - димер, т.е. пара атомов $\mathrm{Cu}$ в окружении одного атома $\mathrm{Sb}_{\mathrm{Zn}}$, коэффициент $a=0$, если этот комплекс нейтральный, $a=1$, если комплекс акцепторный. Это выражение упрощается, если учесть распределение $\mathrm{Cu}$ по различным позициям в решетке $[\mathrm{Cu}]=\left[\mathrm{Cu}_{\mathrm{Zn}}\right]+\left[\mathrm{Cu}_{\mathrm{Sb}}\right]+\left[\mathrm{Cu}_{i}\right]+2\left[\mathrm{Cu}_{2}\right]$, тогда

$$
\begin{aligned}
P= & {[\mathrm{Cu}]-2\left[\mathrm{Cu}_{i}\right]-(2-a)\left[\mathrm{Cu}_{2}\right]-\left[\mathrm{Zn}_{i}\right]-\left[V_{\mathrm{Sb}}\right] } \\
& -\left[\mathrm{Sb}_{\mathrm{Zn}}\right]+\left[V_{\mathrm{Zn}}\right]+\left[\mathrm{Zn}_{\mathrm{Sb}}\right] .
\end{aligned}
$$

В таком виде уравнение нейтральности показывает, какие дефекты вызывают отклонение $P$ от $[\mathrm{Cu}]$, и выделяет группы доноров $\left(\mathrm{Cu}_{i}, \mathrm{Zn}_{i}, V_{\mathrm{Sb}}\right.$ и $\left.\mathrm{Sb}_{\mathrm{Zn}}\right)$, „дополнительных“ акцепторов $\left(V_{\mathrm{Zn}}\right.$ и $\left.\mathrm{Zn}_{\mathrm{Sb}}\right)$ и комплексы $\mathrm{Cu}_{2}$. Для дальнейшего рассмотрения некоторыми слагаемыми можно пренебречь, пользуясь некими общими соображениями. Можно не учитывать доноры $\mathrm{Sb}_{\mathrm{Zn}}$, так как катионная подрешетка перенаселена, а атомов $\mathrm{Sb}$, напротив, не хватает для заселения анионных узлов. При невысоких температурах можно также пренебречь акцепторами $V_{\mathrm{Zn}}$, поскольку при имеющейся концентрации дырок, меньшей полной концентрации меди, концентрация вакансий Zn не может быть значительной. Вакансии цинка, как и вакансии сурьмы, в малой концентрации могут присутствовать, но их основная роль в рассматриваемых образцах сводится не к созданию дополнительных свободных носителей заряда, а к взаимодействию с примесными дефектами, определяющему поведение последних, а следовательно, и суммарный легирующий эффект.

Уравнение нейтральности следует дополнить условием равенства числа узлов в обеих подрешетках, которое записывается как

$$
\left[\mathrm{Cu}_{\mathrm{Zn}}\right]+\left[V_{\mathrm{Zn}}\right]+\left[\mathrm{Sb}_{\mathrm{Zn}}\right]+2\left[\mathrm{Cu}_{2}\right]=\left[\mathrm{Cu}_{\mathrm{Sb}}\right]+\left[\mathrm{Zn}_{\mathrm{Sb}}\right]+\left[V_{\mathrm{Sb}}\right] .
$$

Основу дальнейшей интерпретации составляет наличие достаточно широкого диапазона температур, 565-605 K, в котором, как упоминалось выше, $P=[\mathrm{Cu}]$ (рис. 1). В этих условиях суммой всех слагаемых, кроме $[\mathrm{Cu}]$ в правой части уравнения нейтральности (1), можно пренебречь, а ввиду малости $\left[V_{\mathrm{Zn}}\right]$ и $\left[\mathrm{Zn}_{\mathrm{Sb}}\right]$ можно пренебречь и каждой концентрацией, входящей в уравнение со знаком минус. С учетом (2) это означает, что $\left[\mathrm{Cu}_{\mathrm{Zn}}\right]=\left[\mathrm{Cu}_{\mathrm{Sb}}\right]=(1 / 2)[\mathrm{Cu}]$. Таким образом, примесь $\mathrm{Cu}$ поровну распределена между узлами $\mathrm{Zn}$ и $\mathrm{Sb}$, образуя в обоих случаях акцепторные центры. Общее число узлов, внесенных медью в каждую из подрешеток, равно $(1 / 2)[\mathrm{Cu}]$ и не меняется в исследованном температурном интервале, оно устанавливается при температуре синтеза. Концентрации остальных дефектов в этом диапазоне температур много меньше $(1 / 2)[\mathrm{Cu}] \approx 10^{19} \mathrm{~cm}^{-3}$. Изменения концентрации дырок при других температурах следует связывать с отклонениями от этой базовой дефектной структуры.

При температурах $T>600 \mathrm{~K}$ концентрация дырок растет с температурой, что должно быть связано с появлением дополнительных акцепторов. Единственно возможными дополнительными акцепторами являются антиструктурные дефекты $\mathrm{Zn}_{\mathrm{Sb}}$, которые генерируются по реакции $\mathrm{Zn}_{\mathrm{Zn}}+\mathrm{Cu}_{\mathrm{Sb}} \rightarrow \mathrm{Zn}_{\mathrm{Sb}}+\mathrm{Cu}_{\mathrm{Zn}}$. Реакция не изменяет общее число акцепторов $\mathrm{Cu}$, но превращает нейтральные центры $\mathrm{Zn}_{\mathrm{Zn}}$ в акцепторы $\mathrm{Zn}_{\mathrm{Sb}}$. Эта реакция - обмен местами атомов $\mathrm{Zn}_{\mathrm{Zn}}$ и соседнего $\mathrm{Cu}_{\mathrm{Sb}}$ (у всякого узла $\mathrm{Sb}$, в том числе у занятого атомом $\mathrm{Cu}$, есть 4 соседних атома $Z_{\mathrm{Zn}}$ [9]). Это процесс быстрый, так как происходит на расстояниях порядка межатомных, и его можно представить как: 1) образование 
тесной пары Френкеля $\left.\mathrm{Cu}_{\mathrm{Sb}} \rightarrow \mathrm{Cu}_{i}+V_{\mathrm{Sb}}, 2\right)$ перескок атома $\mathrm{Zn}$ в соседнюю вакансию, $\mathrm{Zn}_{\mathrm{Zn}}+V_{\mathrm{Sb}} \rightarrow \mathrm{Zn}_{\mathrm{Sb}}+V_{\mathrm{Zn}}$, 3) рекомбинация тесной пары Френкеля, $\mathrm{Cu}_{i}+V_{\mathrm{Zn}} \rightarrow$ $\rightarrow \mathrm{Cu}_{\mathrm{Zn}}$. Уравнение нейтральности в этой области температур имеет вид $P=[\mathrm{Cu}]+\left[\mathrm{Zn}_{\mathrm{Sb}}\right]$. Из рис. 1 следует, что $P(710 \mathrm{~K})=2.44 \cdot 10^{19} \mathrm{~cm}^{-3}$, или $\left[\mathrm{Zn}_{\mathrm{Sb}}\right]=$ $=4 \cdot 10^{18} \mathrm{~cm}^{-3}$. Это составляет только $20 \%$ от $[\mathrm{Cu}]$ или $40 \%$ от $\left[\mathrm{Cu}_{\mathrm{Sb}}\right]$.

Ниже $550 \mathrm{~K} P<[\mathrm{Cu}]$, что может быть связано либо с появлением донорных центров, либо с уменьшением концентрации акцепторов за счет образования димеров $\mathrm{Cu}_{2}$. Первый вариант означает увеличение концентрации дефектов $\mathrm{Cu}_{i}$ или $V_{\mathrm{Sb}}$ с понижением температуры, что противоречит обычным представлениям о температурной зависимости таких дефектов и требует введения особых предположений. При этом надо иметь в виду, что, во-первых, относительная потенциальная энергия атомов $\mathrm{Cu}$ в междоузлиях, $\mathrm{Cu}_{i}$, положительная, $\Delta E_{i}>0$, так как $\left[\mathrm{Cu}_{i}\right]<\left[\mathrm{Cu}_{\mathrm{Sb}}\right]$, по крайней мере около $500 \mathrm{~K}$, а во-вторых, $\Delta E_{i}$ должна быстро убывать с уменьшением температуры, чтобы обеспечить рост $\left[\mathrm{Cu}_{i}\right]$ и, следовательно, убывание $P$ с понижением температуры.

Второй вариант означает связывание части атомов $\mathrm{Cu}_{\mathrm{Zn}}$ в димеры при пониженных температурах, $T<550 \mathrm{~K}$, и их распад при повышении температуры. Как димеризация, так и распад димеров - это процессы медленные, требующие перемещения атомов $\mathrm{Cu}$ на расстояния $[\mathrm{Cu}]^{-1 / 3} \approx 4 \cdot 10^{-7}$ см. Уравнение нейтральности в этой области температур принимает вид $P=[\mathrm{Cu}]-(2-a)\left[\mathrm{Cu}_{2}\right]$.

Для $\mathrm{ZnSb}: 0.1 \mathrm{aт} \% \mathrm{Cu}$ имеем: $[\mathrm{Cu}]=2.05 \cdot 10^{19} \mathrm{~cm}^{-3}=$ $=P(580 \mathrm{~K}) ; \quad P(325 \mathrm{~K})=1.67 \cdot 10^{19} \mathrm{~cm}^{-3}, \quad$ что ведет к $\left[\mathrm{Cu}_{2}\right](325 \mathrm{~K})=3.8 \cdot 10^{18} \mathrm{~cm}^{-3}$, если $a=1, \quad$ или $1.9 \cdot 10^{18} \mathrm{~cm}^{-3}$, если $a=0$. Концентрация димеров оказывается на порядок меньше концентрации меди. Концентрация одиночных центров $\left[\mathrm{Cu}_{\mathrm{Zn}}\right]=$ $=(1 / 2)[\mathrm{Cu}]-2\left[\mathrm{Cu}_{2}\right]$ меняется от $(0.28 / 0.65) \cdot 10^{19} \mathrm{~cm}^{-3}$ при $325 \mathrm{~K}$ (для разных $a$ ) до $1.02 \cdot 10^{19} \mathrm{~cm}^{-3}$ при $580 \mathrm{~K}$.

Если при $T>600 \mathrm{~K}$ значения концентраций дефектов считать равновесными (почти нет гистерезиса), то для атомов меди можно применить статистику Больцмана: $\left[\mathrm{Cu}_{\mathrm{Sb}}\right]=\left[\mathrm{Cu}_{\mathrm{Zn}}\right] \exp \left(-\Delta E_{\mathrm{Cu}} / k T\right)$, где $\Delta E_{\mathrm{Cu}}-$ разность потенциальной энергии атомов меди в узлах $\mathrm{Sb}$ и $\mathrm{Zn}$ (рис. 2). При этом $\left[\mathrm{Cu}_{\mathrm{Sb}}\right]$ и $\left[\mathrm{Cu}_{\mathrm{Zn}]}\right.$ связаны соотношением $\left[\mathrm{Cu}_{\mathrm{Sb}}\right]+\left[\mathrm{Cu}_{\mathrm{Zn}}\right]=[\mathrm{Cu}]$ при $\left[\mathrm{Cu}_{i}\right] \approx 0$. Отсюда: $\left[\mathrm{Cu}_{\mathrm{Sb}}\right]=[\mathrm{Cu}] /\left[\left(1+\exp \left(\Delta E_{\mathrm{Cu}} / k T\right)\right]\right.$, $\left[\mathrm{Cu}_{\mathrm{Zn}}\right]=[\mathrm{Cu}] /\left[\left(1+\exp \left(-\Delta E_{\mathrm{Cu}} / k T\right)\right]\right.$.

Равенство $\left[\mathrm{Cu}_{\mathrm{Sb}}\right]=\left[\mathrm{Cu}_{\mathrm{Zn}}\right]=(1 / 2)[\mathrm{Cu}]$ имеет место, когда $\Delta E_{\mathrm{Cu}}=0$, т. е. потенциальные энергии $\mathrm{Cu}_{\mathrm{Sb}}$ и $\mathrm{Cu}_{\mathrm{Zn}}$ равны между собой. Этот случай уже был рассмотрен выше. С ростом температуры $\left[\mathrm{Zn}_{\mathrm{Sb}}\right]$ растет, следовательно, $\left[\mathrm{Cu}_{\mathrm{Sb}}\right]$ убывает, а это означает, что $\Delta E_{\mathrm{Cu}}>0$.

Значения $\Delta E_{\mathrm{Cu}}=f(T)$ можно найти из температурной зависимости концентрации дырок:

$$
\begin{aligned}
P & =[\mathrm{Cu}]+\left[\mathrm{Zn}_{\mathrm{Sb}}\right]=[\mathrm{Cu}]+(1 / 2)[\mathrm{Cu}]-\left[\mathrm{Cu}_{\mathrm{Sb}}\right] \\
& =(3 / 2)[\mathrm{Cu}]-[\mathrm{Cu}] /\left[1+\exp \left(\Delta E_{\mathrm{Cu}} / k T\right)\right],
\end{aligned}
$$

отсюда следует $\Delta E_{\mathrm{Cu}}=k T \ln \{(2 P-[\mathrm{Cu}]) /(3[\mathrm{Cu}]-2 P)\}$.

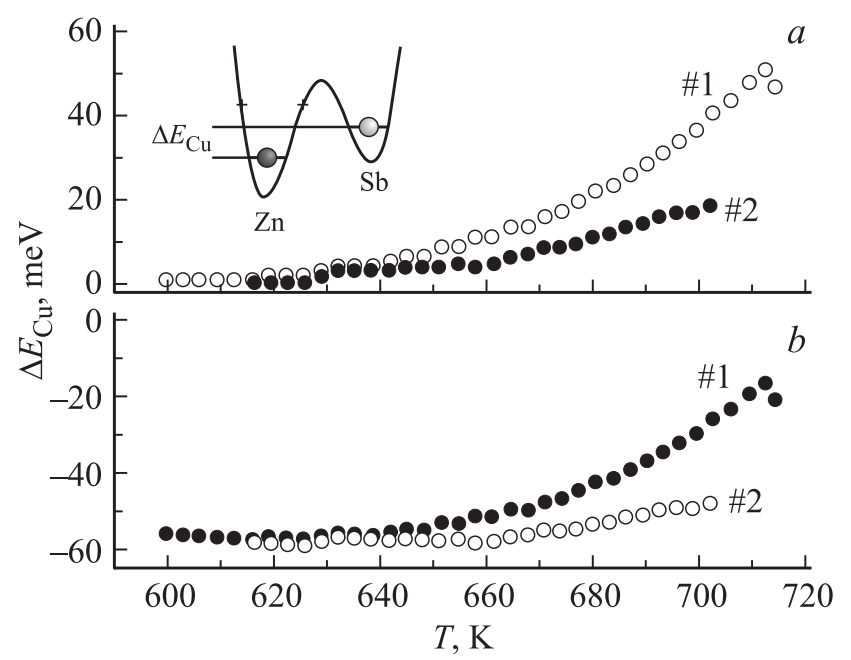

Рис. 2. Потенциальная энергия атома $\mathrm{Cu} \Delta E_{\mathrm{Cu}}$ в узле $\mathrm{Sb}$ относительно ее значения в узле $\mathrm{Zn}$ как функция температуры для образцов $\mathrm{ZnSb}: 0.1 \mathrm{aT} \% \mathrm{Cu}(\# 1), \mathrm{ZnSb}: 0.15 \mathrm{aT} \% \mathrm{Cu}$ (\#2): $a-$ без учета статистических весов состояний, $b-$ с учетом.

Обнаруживается, что разность энергий атома $\mathrm{Cu}$ в узлах $\mathrm{Sb}$ и $\mathrm{Zn}$ растет с повышением температуры. Можно учесть разницу статистических весов состояний $\mathrm{Cu}_{\mathrm{Sb}}$ и $\mathrm{Cu}_{\mathrm{Zn}}$ : переход $\mathrm{Cu}_{\mathrm{Sb}}$ возможен в любой из 3 соседних узлов $\mathrm{Zn}$ (расстояние до четвертого заметно больше, чем до трех других), а переход $\mathrm{Zn}_{\mathrm{Zn}}$ только в один из узлов $\mathrm{Sb}$, в позицию $\mathrm{Cu}_{\mathrm{Sb}}$. Поэтому $\left[\mathrm{Cu}_{\mathrm{Sb}}\right]=(1 / 3)\left[\mathrm{Cu}_{\mathrm{Zn}}\right] \exp \left(-\Delta E_{\mathrm{Cu}} / k T\right)$. В выражении для $\Delta E_{\mathrm{Cu}}$ появляется слагаемое $k T \ln 3$ :

$$
\Delta E_{\mathrm{Cu}}=k T \ln \{(2 P-[\mathrm{Cu}]) /(3[\mathrm{Cu}]-2 P)\}-k T \ln 3 .
$$

В этом приближении оказывается, что положение $\mathrm{Cu}_{\mathrm{Sb}}$ энергетически выгоднее, но равная заселенность $\mathrm{Cu}_{\mathrm{Sb}}$ и $\mathrm{Cu}_{\mathrm{Zn}}$ достигается за счет большего статистического веса последнего. На рис. 2 представлена потенциальная энергия атома $\mathrm{Cu}$ в узле $\mathrm{Sb}$ относительно ее значения в узле $\mathrm{Zn}$ как функция температуры для образца состава $\mathrm{ZnSb}: 0.1 \mathrm{aT} \% \mathrm{Cu}$.

Для $\mathrm{ZnSb}: 0.15 \mathrm{aT} \% \mathrm{Cu}$ (образец \#2) температурная зависимость концентрации дырок (рис. 1) имеет небольшие отличия: плато $P=[\mathrm{Cu}]=3.1 \cdot 10^{19} \mathrm{~cm}^{-3}$ $\left(\left[\mathrm{Cu}_{\mathrm{Sb}}\right]=\left[\mathrm{Cu}_{\mathrm{Zn}}\right]=(1 / 2)[\mathrm{Cu}]\right)$ смещено в область высоких температур на $30 \mathrm{~K}$; концентрация дополнительных акцепторов при $700 \mathrm{~K}$ меньше, $\left[\mathrm{Zn}_{\mathrm{Sb}}\right]=1.25 \cdot 10^{18} \mathrm{~cm}^{-3}$; убыль концентрации дырок при $325 \mathrm{~K}, 7 \cdot 10^{18} \mathrm{~cm}^{-3}$, примерно в 2 раза больше, чем для образца \#1. Для объяснения отличий по той же схеме была рассчитана $\Delta E_{\mathrm{Cu}}$ (рис. 2). Оказалось, что $\Delta E_{\mathrm{Cu}}$ зависит от концентрации примеси и существенно меньше, чем для образца $\mathrm{ZnSb}: 0.1 \mathrm{aт} \% \mathrm{Cu}$. Это объясняет уменьшение доли $\left[\mathrm{Zn}_{\mathrm{Sb}}\right]$ : из-за меньшего перепада энергии атомы $\mathrm{Cu}$ менее охотно покидают узлы Sb. C этим же связано смещение плато $P=[\mathrm{Cu}]$ к более высоким температурам.

Используя схожее соотношение $\left[\mathrm{Zn}_{\mathrm{Sb}}\right]=(1 / 3)\left[\mathrm{Zn}_{\mathrm{Zn}}\right]$ $\times \exp \left(-\Delta E_{Z n} / k T\right)$, можно найти разность потенциальной энергии атома $\mathrm{Zn}$ в узлах $\mathrm{Sb}$ и $\mathrm{Zn}\left(E_{\mathrm{Sb}}-E_{\mathrm{Zn}}\right)$. 


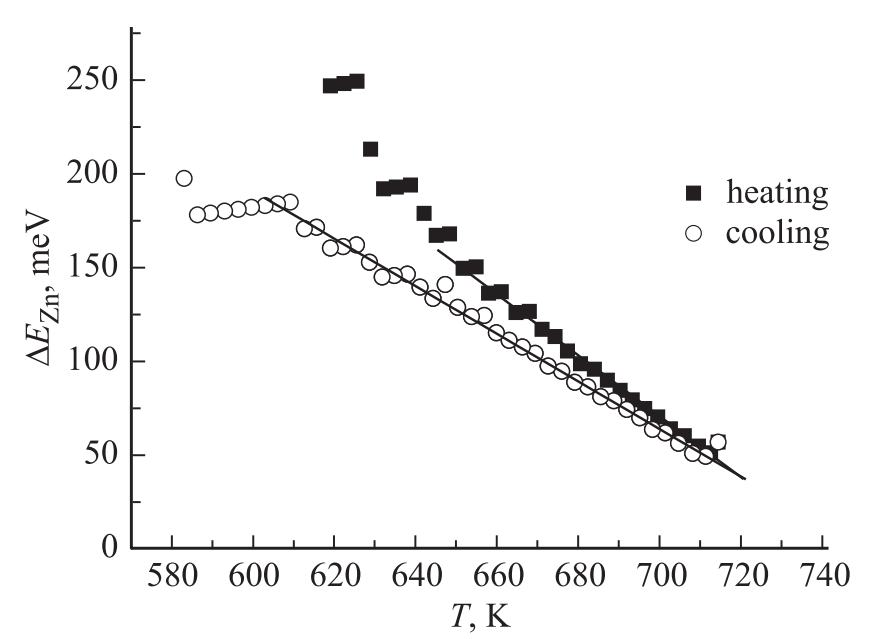

Рис. 3. Потенциальная энергия атома $\mathrm{Zn} \Delta E_{\mathrm{Zn}}$ в узле $\mathrm{Sb}$ относительно ее значения в собственном узле как функция температуры для $\mathrm{ZnSb}: 0.1 \mathrm{aT} \% \mathrm{Cu}$ (\#1) с учетом статистических весов состояний.

Речь идет не обо всех атомах $\mathrm{Zn}_{\mathrm{Zn}}$, а только о тех, которые имеют в соседях атомы $\mathrm{Cu}_{\mathrm{Sb}}$. Множитель $1 / 3$, как и выше, означает, что статистический вес атома $\mathrm{Zn}_{\mathrm{Zn}}$ в 3 раза больше, чем атома $\mathrm{Zn}_{\mathrm{Sb}}$.

Условие постоянства числа атомов: $\left[\mathrm{Zn}_{\mathrm{Sb}}\right]+\left[\mathrm{Zn}_{\mathrm{Zn}}\right]=$ $=3[\mathrm{Cu}] / 2$ (число атомов $\mathrm{Zn}_{\mathrm{Zn}}$ в 3 раза больше числа узлов $\mathrm{Cu}_{\mathrm{Sb}}=(1 / 2)[\mathrm{Cu}]$, в которые они переходят $)$,

$$
\left(3[\mathrm{Cu}] / 2-\left[\mathrm{Zn}_{\mathrm{Sb}}\right]\right) /\left[\mathrm{Zn}_{\mathrm{Sb}}\right]=3 \exp \left(\Delta E_{\mathrm{Zn}} / k T\right) .
$$

Источником дополнительных дырок являются атомы $\mathrm{Zn}_{\mathrm{Sb}}:\left[\mathrm{Zn}_{\mathrm{Sb}}\right]=P-[\mathrm{Cu}]$,

$$
\begin{gathered}
(5[\mathrm{Cu}] / 2-P) /(P-[\mathrm{Cu}])=3 \exp \left(\Delta E_{\mathrm{Zn}} / k T\right), \\
\Delta E_{\mathrm{Zn}}=k T \ln \{(5[\mathrm{Cu}]-2 P) /(P-[\mathrm{Cu}])\}-k T \ln 6 .
\end{gathered}
$$

Вычисленные значения $\Delta E_{\mathrm{Zn}}$ представлены на рис. 3 . Они различаются для нагревания и охлаждения, что указывает на неравновесное значение концентрации дефектов. Наблюдается спад $\Delta E_{\mathrm{Zn}}$ с ростом температуры. И при нагреве, и при охлаждении кривые имеют хорошо выраженные линейные участки. Температурная зависимость $\Delta E_{\mathrm{Zn}}$ при охлаждении ближе к линейной, что, повидимому, говорит о большей близости к равновесию. Это можно объяснить меньшей высотой барьера со стороны атома $\mathrm{Zn}_{\mathrm{Sb}}$ по сравнению с высотой от атома $\mathrm{Zn}_{\mathrm{Zn}}$.

Аналогичный расчет для образца $\mathrm{ZnSb}: 0.15 \mathrm{aT} \% \mathrm{Cu}$ дал несколько большее значение для $\Delta E_{\mathrm{Zn}}$, объяснив тем самым появление атомов $\mathrm{Zn}_{\mathrm{Sb}}$ при более высоких температурах, и, кроме того, показал чуть более слабую зависимость $\Delta E_{\mathrm{Zn}}$ от температуры.

Значения концентрации димеров $\left[\mathrm{Cu}_{2}\right]$ определяются легко из уравнения электронейтральности $P=[\mathrm{Cu}]-$ $-(2-a)\left[\mathrm{Cu}_{2}\right]$. Однако измеренные значения $P$ являются неравновесными, а равновесные можно получить из условия равенства скоростей генерации и распада димеров:

$$
\alpha\left([\mathrm{Cu}]-2\left[\mathrm{Cu}_{2}\right]_{p}\right)^{2}=\beta\left[\mathrm{Cu}_{2}\right]_{p} .
$$

Индекс ,p“ при $\left[\mathrm{Cu}_{2}\right]$ означает равновесную концентрацию при данной температуре, а $\alpha$ и $\beta-$ константы скоростей генерации и распада димера, функции температуры. Обозначая $c_{p}=\left[\mathrm{Cu}_{2}\right]_{p} /[\mathrm{Cu}]$ как безразмерную равновесную концентрацию $\left[\mathrm{Cu}_{2}\right]$ и $q=(\beta / \alpha) /[\mathrm{Cu}]$ как безразмерное отношение констант скоростей распада и генерации димеров, равенство (3) в безразмерном виде можно записать в виде $1-4 c_{p}+4 c_{p}^{2}=c_{p} q$. Левая часть этого равенства достигает минимума, равного 0 (при $q=0)$, когда $c_{p}=0.5,\left[\mathrm{Cu}_{2}\right]_{p}=(1 / 2)[\mathrm{Cu}]$, а физический смысл имеют только $c_{p}<0.5$, так как $\left[\mathrm{Cu}_{2}\right]_{p}$ не может быть больше $(1 / 2)[\mathrm{Cu}]$. В итоге равенство (3) сводится к квадратному уравнению $4 c_{p}^{2}-(4+q) c_{p}+1=0$, решение которого дает значение $c_{p}=\left[\mathrm{Cu}_{2}\right]_{p} /[\mathrm{Cu}]$ как функцию параметра $q$. Чтобы воспользоваться этим решением, надо знать отношение $q$; из (3) получаем

$$
q=\left([\mathrm{Cu}]-2\left[\mathrm{Cu}_{2}\right]_{p}\right)^{2} /\left(\left[\mathrm{Cu}_{2}\right]_{p}[\mathrm{Cu}]\right) .
$$

Непосредственно воспользоваться этим соотношением невозможно, так как измеренные значения $\left[\mathrm{Cu}_{2}\right]$ неравновесные. Однако можно получить функцию $q=f(T)$, если задаться ее видом, например, воспользоваться формулой Аррениуса $q=A \exp (\Delta / k T)$, далее допустить на температурной зависимости концентрации существование почти равновесного участка в области температур ниже $580 \mathrm{~K}$, для него из (3) найти $q=f(T)$, проверить выполнение закона Аррениуса, определить значения констант и затем экстраполировать $q=A \exp (\Delta / k T)$ на температурный диапазон, в котором условия очевидно неравновесные. Несмотря на отсутствие строгого обоснования такой процедуры, расчеты по ней были проведены. В качестве почти равновесного участка был принят диапазон $450-510 \mathrm{~K}$ в режиме охлаждения, в котором при $510 \mathrm{~K}$ отклонения $\left[\mathrm{Cu}_{2}\right]$ от равновесных малы. Для нахождения $\left[\mathrm{Cu}_{2}\right]$ и $q$ использовали измеренные значения концентрации $P=[\mathrm{Cu}]-(2-a)\left[\mathrm{Cu}_{2}\right]$. Расчеты проводились в 4 вариантах (см. таблицу): для двух значений $a(0$ и 1$)$ и двух значений концентрации меди, служившей источником атомов для димеров. Первое значение концентрации меди, равное $[\mathrm{Cu}]$, предполагает, что димеры образуются из атомов обеих подрешеток $\mathrm{Cu}_{\mathrm{Sb}}$ и $\mathrm{Cu}_{\mathrm{Zn}}$, что осложнено образованием $V_{\mathrm{Sb}}$, но зато дает дополнительный донор. Второе значение есть $(1 / 2)[\mathrm{Cu}]$, если источником служат только атомы $\mathrm{Cu}_{\mathrm{Zn}}$, что требует замены $[\mathrm{Cu}]$ на $(1 / 2)[\mathrm{Cu}]$ при переходе от $c_{p}$ к $\left[\mathrm{Cu}_{2}\right]_{p}$. Результаты для $q$ при $T<510 \mathrm{~K}$, которые должны соотноситься со значениями $A \exp (\Delta / k T)$, представлены на рис. 4 для разных вариантов расчета (см. таблицу) в полулогарифмическом масштабе. Определены параметры линейных участков при $430-510 \mathrm{~K}$ (см. таблицу) и затем рассчитаны температурные зависимости $c_{p},\left[\mathrm{Cu}_{2}\right]_{p}$ и далее $P$ (рис. 5). 
Данные для расчета равновесных значений концентрации $P$ при охлаждении для образца \# 1

\begin{tabular}{|c|c|c|c|c|}
\hline \multirow{3}{*}{ Результаты } & \multicolumn{4}{|c|}{ Вариант расчета } \\
\hline & 1 & 2 & 3 & 4 \\
\hline & {$[\mathrm{Cu}], a=1$} & $(1 / 2)[\mathrm{Cu}], a=0$ & $(1 / 2)(\mathrm{Cu}), a=1$ & {$[\mathrm{Cu}], a=0$} \\
\hline $\begin{array}{c}\text { Линейная зависимость } \\
\ln q=f(x) \text { на рис. } 4\end{array}$ & \multicolumn{2}{|c|}{$y=-7100 x+19.1$} & $y=-7600 x+19.4$ & $y=-6700 x+19.0$ \\
\hline$\Delta / k$ & \multicolumn{2}{|c|}{$7100 \mathrm{~K}$} & $7600 \mathrm{~K}$ & $6700 \mathrm{~K}$ \\
\hline$A$ & \multicolumn{2}{|c|}{$1.95 \cdot 10^{8}$} & $2.66 \cdot 10^{8}$ & $1.78 \cdot 10^{8}$ \\
\hline$\left[\mathrm{Cu}_{2}\right]$ & {$[\mathrm{Cu}] c_{p}$} & $(1 / 2)[\mathrm{Cu}] c_{p}$ & $(1 / 2)[\mathrm{Cu}] c_{p}$ & {$[\mathrm{Cu}] c_{p}$} \\
\hline $\begin{array}{c}P, \text { номер кривой } P(T) \\
\text { на рис. } 5\end{array}$ & $\begin{array}{c}{[\mathrm{Cu}]-\left[\mathrm{Cu}_{2}\right]} \\
1 / 2\end{array}$ & $\begin{array}{c}{[\mathrm{Cu}]-2\left[\mathrm{Cu}_{2}\right]} \\
1 / 2\end{array}$ & $\begin{array}{c}{[\mathrm{Cu}]-\left[\mathrm{Cu}_{2}\right]} \\
3\end{array}$ & $\begin{array}{c}{[\mathrm{Cu}]-2\left[\mathrm{Cu}_{2}\right]} \\
4\end{array}$ \\
\hline
\end{tabular}

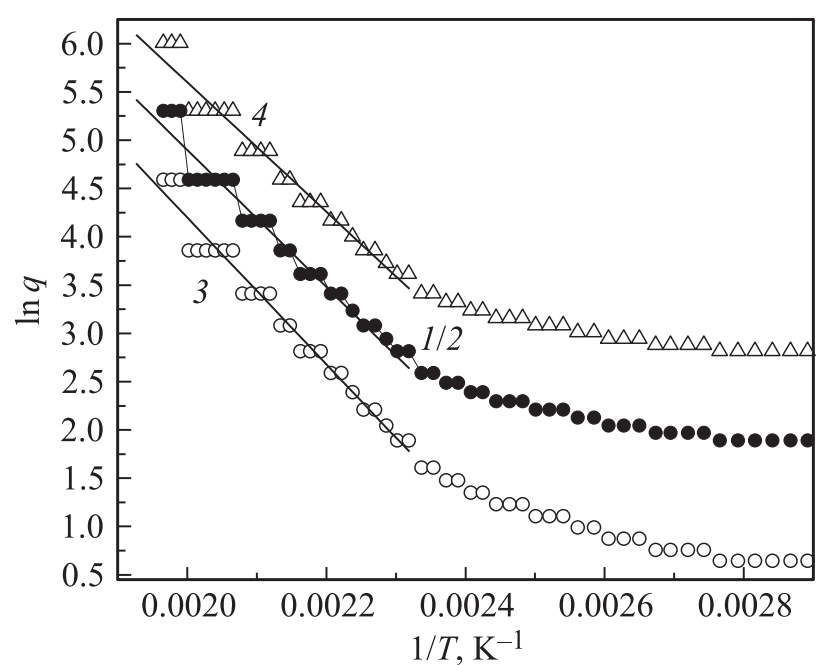

Рис. 4. Зависимость безразмерного отношения констант скоростей распада и генерации димеров $q=\left([\mathrm{Cu}]-2\left[\mathrm{Cu}_{2}\right]_{p}\right)^{2} /$ $\left(\left[\mathrm{Cu}_{2}\right]_{p}[\mathrm{Cu}]\right)$ от обратной температуры в полулогарифмическом масштабе для образца \#1 в вариантах расчета 1-4 (см. таблицу), номера кривых соответствуют номерам вариантов расчета.

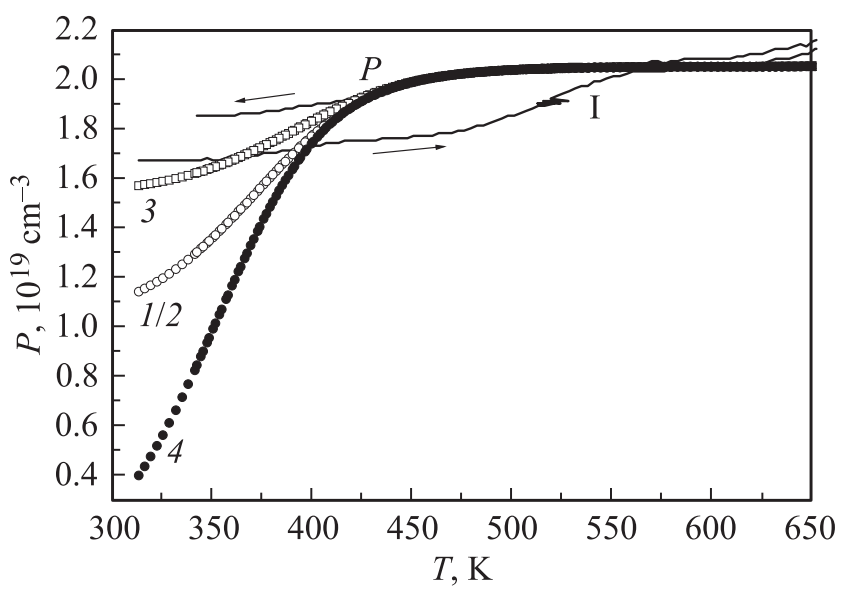

Рис. 5. Равновесные значения концентрации дырок при охлаждении в цикле I, полученные в вариантах расчета 1-4 (см. таблицу), в сопоставлении с экспериментальными данными, номера кривых соответствуют номерам вариантов расчета.
Кривые $\ln q$, значения $q$ и $c_{p}$ в вариантах $(1 / 2)[\mathrm{Cu}]$, $a=0$ и $[\mathrm{Cu}], a=1$ совпадают, а в двух других различаются; к первым двум на рис. 5 относится линия 1/2, к вариантам 3 и 4 - соответственно 3 и 4. По-видимому, вариант 3 , т.е. простой акцептор $\mathrm{Cu}_{2}$, получающийся только из атомов $\mathrm{Cu}_{\mathrm{Zn}}$, соответствует экспериментальным данным. Полученное согласие не избавляет, однако, от необходимости экспериментального определения равновесной концентрации дырок. Решению этой задачи для комнатной температуры способствовало проведение дополнительных циклов изменения температуры на образце $\mathrm{ZnSb}: 0.1 \mathrm{aT} \% \mathrm{Cu}$, описанное далее.

\section{3. Экспериментальные данные для повторных термоциклов и их обсуждение}

Температурные зависимости концентрации дырок $P$ и подвижности $\mu$, полученные в серии циклов в режиме 300-700-300 K на том же образце $\mathrm{ZnSb}: 0.1 \mathrm{aт} \% \mathrm{Cu}$, представлены на рис. 6. Цикл I проводился сразу после приготовления отожженного и закаленного образца, а последующие циклы II-VIII различались временем выдержки при комнатной температуре, предваряющей начало измерений, т. е. длительностью предварительного низкотемпературного отжига: для II - это три месяца, III проводился без разрыва от II и начинался от $77 \mathrm{~K}$; циклы IV и V шли с большим предварительным отжигом образца при $300 \mathrm{~K}$, VI и VII перед началом нагревания находились при $300 \mathrm{~K}$ в течение 20 и 13 дней соответственно, заключительный в настоящее время VIII цикл проводился через 1/2 суток после цикла VII. Самый длительный отжиг разделял циклы IV и V и длился 1.75 года. Полное время работы с данным образцом фактически дошло до трех лет. Надо отметить, что свойства образца при охлаждении, когда предшествующее нагревание убирает негативное влияние низкотемпературных дефектов, разное в разных циклах, проявляют высокую устойчивость (рис. 6).

Ход кривых нагревания до $T \approx 540 \mathrm{~K}$ заметно зависит от длительности низкотемпературного отжига, с ее уве- 


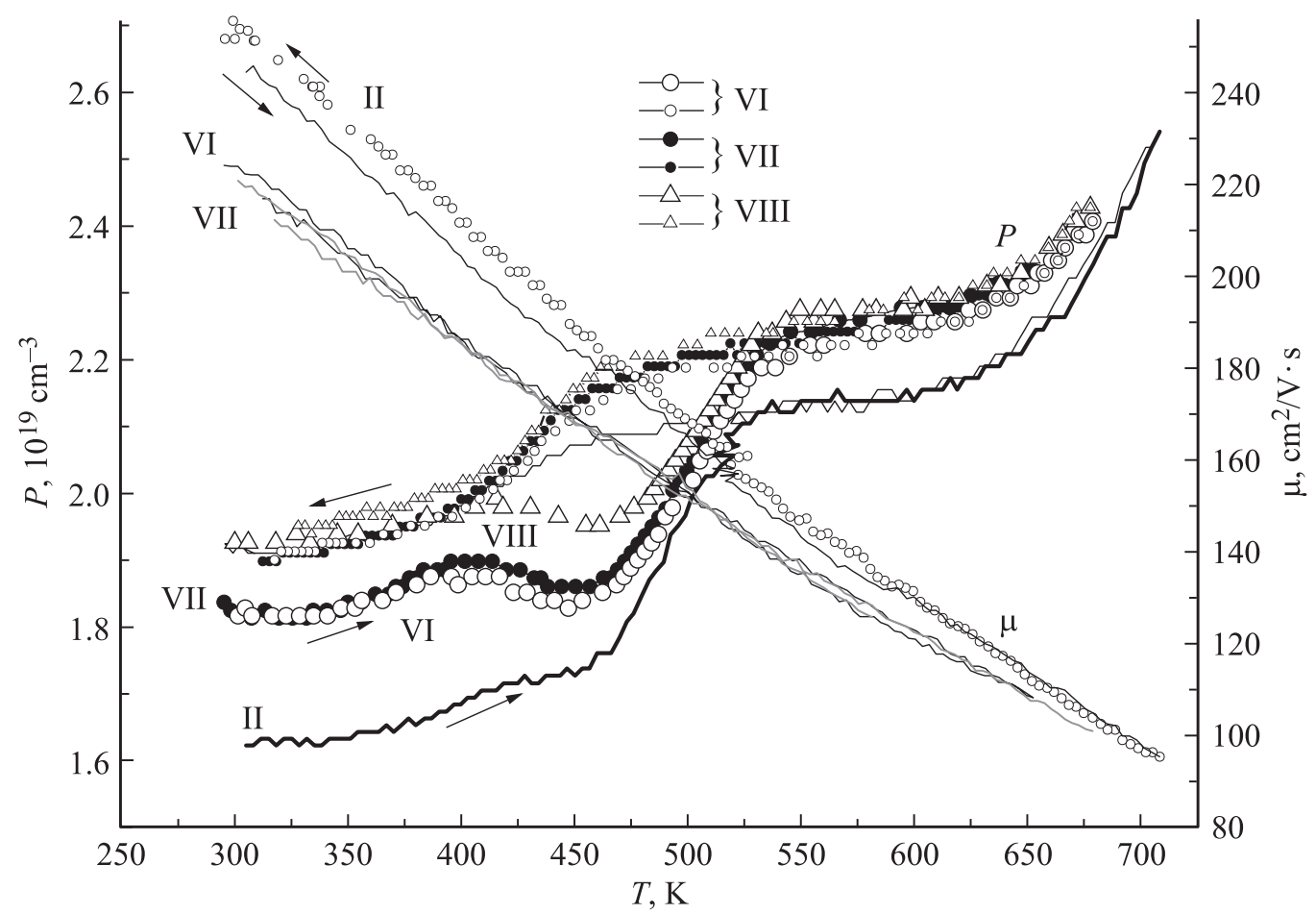

Рис. 6. Температурные зависимости концентрации $P$ и подвижности $\mu$ в образце $\mathrm{ZnSb}: 0.1 \mathrm{aT} \% \mathrm{Cu}$ для повторных циклов с разной длительностью низкотемпературного отжига $(300 \mathrm{~K})$, предваряющего начало измерений: для цикла II - 3 месяца, для циклов VI, VII - соответственно 20 и 13 дней, для цикла VIII - 0.5 суток. Самый длительный отжиг при $300 \mathrm{~K}$ разделял циклы IV и $\mathrm{V}$ и длился 1.75 года, $P(T)$ для цикла $\mathrm{V}$ представлена на рис. 1. Полное время работы с образцом - 3 года. Обозначения: прямые ветви $P(T)$ (нагрев) в циклах VI, VII, VIII - крупные значки; обратные ветви - мелкие; в цикле II — соответственно толстая и тонкая линии. Кривые $\mu(T)$ для циклов VI, VII практически неразличимы.

личением уменьшается начальная концентрация дырок. Самое низкое значение холловской концентрации, которое можно принять близким к равновесному, получено в цикле $\mathrm{V}$ и равно $1.2 \cdot 10^{19} \mathrm{~cm}^{-3}$, что составляет $\sim 60 \%$ от полной акцепторной активности атомов меди, потеря $40 \%$ связана с образованием димеров; их число в предположении, что это простые акцепторы, $a=1$, равно $4 \cdot 10^{18} \mathrm{~cm}^{-3}$. Снижение $P$, естественно, уменьшает и электропроводность $\sigma$. На рис. 7 представлены кривые $\sigma(T)$, полученные совершенно в разных условиях, но качественно и количественно некоторые из них удивительно согласуются, подтверждая, что пониженные температуры уменьшают $\sigma$ значительно сильнее, чем высокие $T$ (имеются в виду кривые 5 и 10).

Вторая особенность состоит в том, что в зависимости от длительности низкотемпературного отжига распад димеров можно отождествлять либо с двумя процессами разной интенсивности (циклы II, VI, VII), либо с одним (циклы IV, V), который заканчивается при температуре $T \approx 540 \mathrm{~K}$, одинаковой для всех повторных циклов. Однако концентрация, соответствующая этой температуре, не постоянна, а непрерывно, хотя и слабо, растет; например, это хорошо видно из сопоставления кривых $P(T)$ для циклов I и V на рис. 1. В цикле I и VIII данные концентрации различаются на 11\%. В повторных циклах рассматриваемая концентрация дырок уже не равна $[\mathrm{Cu}]$, а определяется еще и дополнительным типом акцепторов, концентрация которых возрастает от цикла к циклу. Дополнительным типом акцепторов

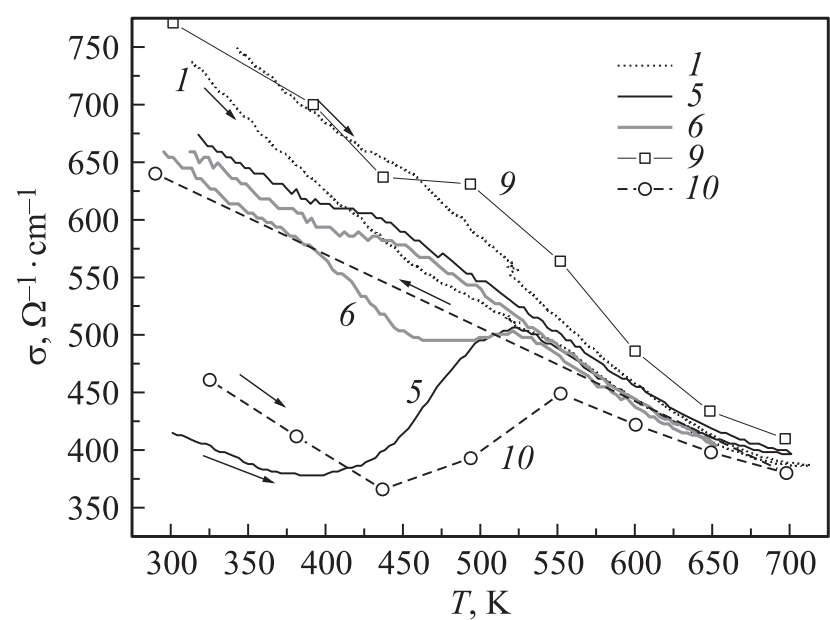

Рис. 7. Температурное изменение электропроводности в циклах I, V и VI (1,5,6 соответственно) с разным предварительным отжигом при комнатной температуре для образца $\mathrm{ZnSb}: 0.1 \mathrm{ar} \% \mathrm{Cu}$, а также для массивного образца того же состава, приготовленного по обычной технологии без дополнительной термообработки (9) и после 1500 -часового отжига в перепаде температур $40-400^{\circ} \mathrm{C}(10)$. 


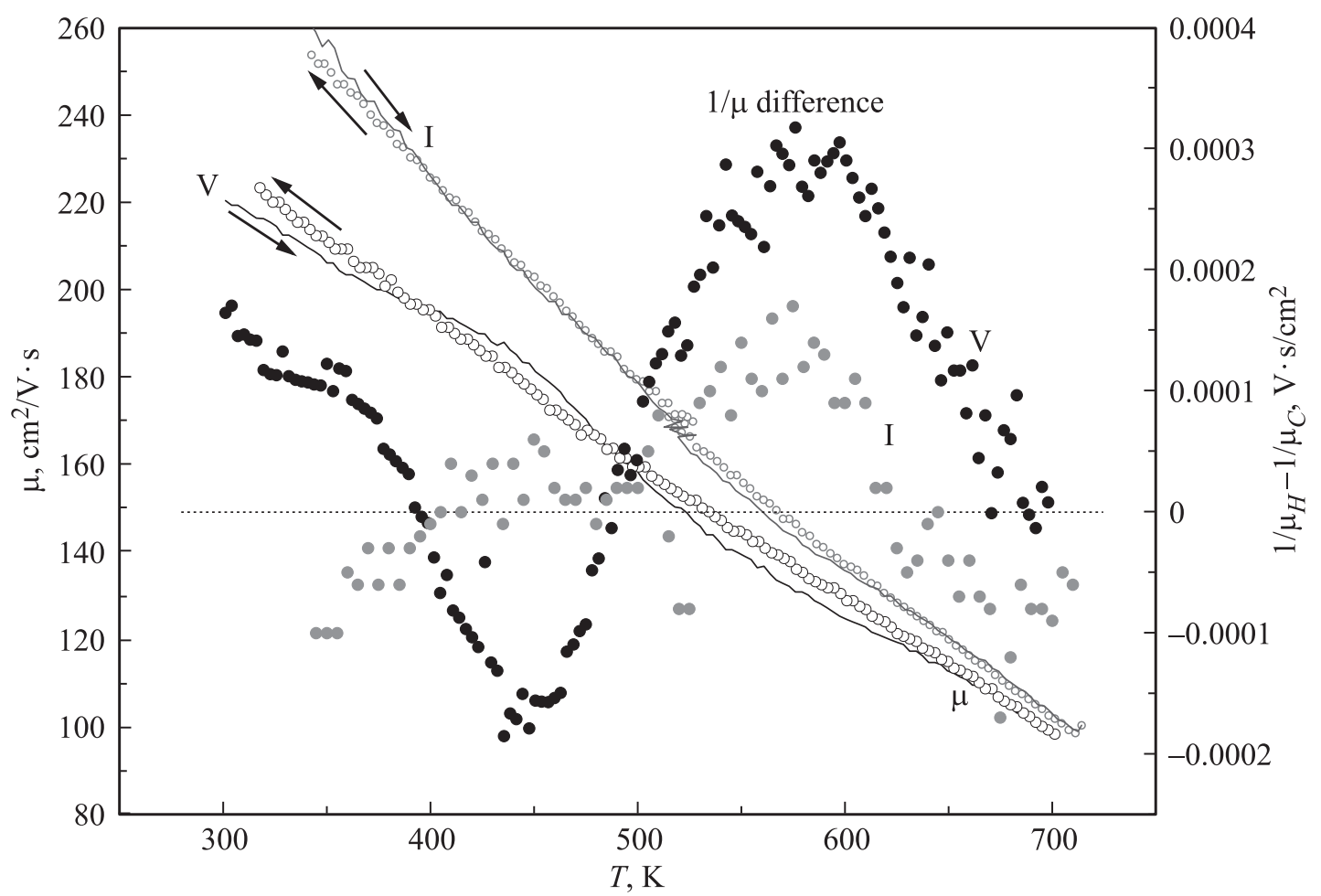

Рис. 8. Сопоставление для циклов I и $\mathrm{V}$ кривых подвижности $\mu$ и разности обратных подвижностей при одной и той же температуре нагревания $\left(1 / \mu_{H}\right)$ и охлаждения $\left(1 / \mu_{C}\right)$. Сопоставление холловских концентраций для этих циклов дано на рис. 1.

при высоких температурах, как было установлено выше, является антиструктурный цинк $\mathrm{Zn}_{\mathrm{Sb}}$, поэтому в данном случае, когда мы имеем дело с повторными циклами, можно предположить, что образование этих дефектов начинается раньше, являясь ответом на уменьшение концентрации одиночных центров $\mathrm{Cu}_{\mathrm{Zn}}$ при образовании димеров, влекущее за собой нарушение установившегося при более высоких температурах равновесия между $\left[\mathrm{Cu}_{\mathrm{Zn}}\right]$ и $[\mathrm{Cu} \mathrm{Sb}]$. Полученные в результате начавшейся реакции $\mathrm{Zn}_{\mathrm{Zn}}+\mathrm{Cu}_{\mathrm{Sb}} \rightarrow \mathrm{Zn}_{\mathrm{Sb}}+\mathrm{Cu}_{\mathrm{Zn}}$ новые порции атомов $\mathrm{Cu}_{\mathrm{Zn}}$ вновь создают димеры и т.д., образовавшаяся при низких температурах концентрация $\left[\mathrm{Zn}_{\mathrm{Sb}}\right]_{0}$ сохраняется до температур $\sim 540 \mathrm{~K}$. Таким образом, к началу высокотемпературного участка на температурной зависимости концентрация дырок $P$ имеет уже более высокое значение, равное $P=[\mathrm{Cu}]+\left[\mathrm{Zn}_{\mathrm{Sb}}\right]_{0}$.

Для дальнейшего обсуждения этой идеи полезно обратиться к подвижности и сравнить не только обе ветви $\mu(T)$ для разных циклов, например I и $\mathrm{V}$, но и разности обратных значений $\mu$ при одной и той же температуре для нагревания и охлаждения в этих циклах (рис. 8). Последнее позволяет определить, в каких диапазонах температур усиливается рассеяние на дефектах с циклами и какое отношение к этому усилению имеют основные типы дефектов, рассмотренные выше. Ситуация с $\mu$ и разностью $1 / \mu$ при $T \leq 484 \mathrm{~K}$ на рис. 8 не вызывает вопросов, все определяется соотношением влияния на $\mu$ концентраций дырок и низкотемпературных дефектов. Интереснее другое: значение $P=2.05 \cdot 10^{19} \mathrm{~cm}^{-3}$ в обоих циклах достигается при разных температурах нагревания, 510 (для цикла $\mathrm{V}$ кривая $P(T)$ приведена на рис. 1) и $566 \mathrm{~K}$, значения разности $1 / \mu$ при этих $T$ близки; однако если в цикле I с ростом $T$ выше $566 \mathrm{~K}$ рассеяние падает и при $\sim 625 \mathrm{~K}$ разность $1 / \mu$ обращается в 0 , то в цикле $\mathrm{V}$ картина принципиально иная после $510 \mathrm{~K}$ рассеяние продолжает так же интенсивно расти, достигая максимума при $P=2.21 \cdot 10^{19} \mathrm{~cm}^{-3}$ и практически при той же $T \approx 566 \mathrm{~K}$. Так же растянут по температуре и спад рассеяния в этом цикле, продолжающийся вплоть до $\sim 680$ К. Качественное сходство и количественные различия хорошо видны при сопоставлении кривых $\mu(T)$ для обоих циклов (рис. 8).

Затем интерес представляют процессы выхода в междоузлия атомов $\mathrm{Cu}$ из обеих подрешеток. Когда узел покидает атом $\mathrm{Cu}_{\mathrm{Sb}}$, снижается не только $\mu$, но и $P$; когда это случается с атомоми $\mathrm{Cu}_{\mathrm{Zn}}$, акцепторное действие остается прежним благодаря появлению дополнительных акцепторов $V_{\mathrm{Zn}}$. Это обстоятельство важно иметь в виду при описании взаимодействия атомов $\mathrm{Zn}$ и антиструктурной меди. Вариант $\mathrm{Zn}_{\mathrm{Zn}}+\mathrm{Cu}_{\mathrm{Sb}} \rightarrow \mathrm{Zn}_{\mathrm{Sb}}+\mathrm{Cu}_{i}+V_{\mathrm{Zn}}$ не изменяет количественного результата, но качественный вывод оказывается другим. Возможно, этот вариант и будет иметь отношение к начальному процессу образования акцепторов $\mathrm{Zn}_{\mathrm{Sb}}$, с этим же процессом может быть связано обнаруженное усиление рассеяния на дефектах, сопровождающее рост концентрации дырок в диапазоне распада димеров. 
Межузельные атомы $\mathrm{Cu}_{i}$ в сочетании с $V_{\mathrm{Sb}}$, возможно, тоже имеют отношение к наблюдаемым изменениям в $P(T)$, поскольку в циклах VI-VIII нагревание в интервале 400-465 К вызывает не рост, а падение концентрации дырок (рис. 6). Не исключено, что фаза с участием доноров и собственных акцепторов предваряет более низкотемпературный процесс димеризации, создавая для него благоприятные условия.

\section{4. Заключение}

В дефектной структуре образца, подвергающегося повторяющейся процедуре термоциклирования в режиме 300-700-300 K, происходят изменения, связанные с медленными процессами образования и распада димеров $\mathrm{Cu}_{2}$ и нарушением равновесия в заселенности подрешеток $\mathrm{Zn}$ и $\mathrm{Sb}$ примесными атомами меди. Эти изменения обнаруживаются в модификации температурных зависимостей холловской концентрации дырок $P(T)$, характер которой не изменяется от цикла к циклу, а величина отклонений от $P(T)$ для первичного цикла возрастает. К таким отклонениям относятся понижение концентрации дырок при температурах $<500 \mathrm{~K}$ и ее увеличение при повышенных температурах. Влияние на электропроводность и подвижность изменений в микроструктуре дефектов более устойчивое и заметнее сказывается на величине ZT. Это легко понять, если иметь в виду отмеченное выше обстоятельство, согласно которому одно и то же изменение $P$ можно реализовать за счет разной микроструктуры дефектов, а это означает, что рассеяние носителей тока в таких случаях может значительно различаться. Не случайно, что во всех циклах электропроводность всегда ниже ее значений для цикла I (рис. 7), это характерно и для подвижности (рис. 6). Можно полагать, что устойчивая, температурно-определяемая микроструктура дефектов в данном материале обеспечивает рост концентрации носителей тока с температурой, сосуществующий с определенной величиной температурного вклада дефектов в рассеяние. Чтобы снижение $\sigma$ не выходило за рамки допустимого, желательно ограничивать диапазон рабочих температур, исключая из него охлаждение материала ниже $100-150^{\circ} \mathrm{C}$. При температурах $450-700 \mathrm{~K}$ данный состав $\mathrm{ZnSb}$ может эффективно и стабильно работать тысячи часов.

\section{Список литературы}

[1] Л.В. Прокофьева, П.П. Константинов, А.А. Шабалдин, Д.А. Пшенай-Северин, А.Т. Бурков, М.И. Федоров. ФТП, 48 (12), 1611 (2014).

[2] Л.В. Прокофьева, М.И. Федоров, А.А. Шабалдин, П.П. Константинов. В сб.: Термоэлектрики и их применения, под ред. М.И. Федорова, Л.Н. Лукьяновой (СПб., ФТИ им. А.Ф. Иоффе РАН, 2015) с. 367.

[3] C. Okamura, T. Ueda, K. Hasezaki. Mater. Trans., 51 (5), 860 (2010).
[4] D. Benson, O.F. Sankey, U. Häussermann. Phys. Rev. B, 84, 125211 (2011).

[5] K. Valset, P.H.M. Böttger, J. Taftø, T.G. Finstad. J. Appl. Phys., 111 (2), 023703 (2012).

[6] D.-B. Xiong, N.L. Okamoto, H. Inui. Scripta Mater., 69, 397 (2013).

[7] M.I. Fedorov, L.V. Prokof'eva, D.A. Pshenay-Severin, A.A. Shabaldin, P.P. Konstantinov. J. Electron. Mater., 43, 2314 (2014).

[8] М.И. Федоров, Л.В. Прокофьева, Ю.И. Равич, П.П. Константинов, Д.А. Пшенай-Северин, А.А. Шабалдин. ФТП, 48 (4), 448 (2014).

[9] Q. Guo, S. Luo. Funct. Mater. Lett., 8 (2), 1550028 (2015).

Редактор Л.В. Шаронова

\section{Temperature dependences of the atomic structure and electrical activity of defects in thermoelectric ZnSb weakly doped with $\mathrm{Cu}$}

\author{
L.V. Prokofieva, F.S. Nasredinov, P.P. Konstantinov, \\ A.A. Shabaldin \\ ${ }^{1}$ loffe Institute, \\ 194021 St. Petersburg, Russia \\ ${ }^{2}$ Peter the Greate State Polytechnical University, \\ 195251 St. Petersburg, Russia
}

\begin{abstract}
The model selection problem is solved for the temperature dependence description for the defect microstructure in high-performance thermoelectric $\mathrm{ZnSb}$ with $0.1 \mathrm{at} \% \mathrm{Cu}$. The temperature dependences of charge carrier concentration and mobility for the first thermal cycle I $300-700-300 \mathrm{~K}$ are analysed, considering distinctive features of the crystal structure and covalent nature of the chemical bond in $\mathrm{ZnSb}$. The basic defect structure (the temperature interval $560-605 \mathrm{~K}$ ) is a state when all the impurity atoms are distributed equally among the sites of the both sublattices and act as acceptors, the number of intrinsic defects of both acceptor and donor types is much less. Their action becomes remarkable when the temperature exceeds the limits of the range cited above. At $T>605 \mathrm{~K}$ an extra acceptors $\mathrm{Zn}_{\mathrm{Sb}}$ appear; on cooling below $550 \mathrm{~K} \mathrm{Cu}_{2}$ dimers are formed: the impurity electrical activity decreases. The dimer decay on heating gives rise to growing concentration up to the value of saturation in the range cited above. Additional thermal cycles II-VIII on the same sample were conducted, some discovered changes in the temperature curves of the properties under study are discussed in the framework of the model considered.
\end{abstract}

